\title{
Management of Metastatic Disease in Campania (MAMETIC): Epidemiologic Data and Palliative Radiotherapy in an Italian Region. Study Protocol
}

Rossella DI FRANCO ( $\nabla$ r.difranco@istitutotumori.na.it )

Istituto Nazionale Tumori IRCCS Fondazione Pascale https://orcid.org/0000-0002-6918-2178

Marco Cascella

Istituto Nazionale Tumori IRCCS Fondazione Pascale

Mario Fusco

Cancer Registry Unit, ASL NA3 SUD

Valentina Borzillo

Istituto Nazionale Tumori IRCCS Fondazione Pascale

Piera Ferraioli

Istituto Nazionale Tumori IRCCS Fondazione Pascale

\section{Esmeralda Scipilliti}

Istituto Nazionale Tumori IRCCS Fondazione Pascale

Emma D'Ippolito

Istituto Nazionale Tumori IRCCS Fondazione Pascale

Angela Petito

Istituto Nazionale Tumori IRCCS Fondazione Pascale

Arturo Cuomo

Istituto Nazionale Tumori IRCCS Fondazione Pascale

Sandro Pignata

Istituto Nazionale Tumori IRCCS Fondazione Pascale

Marilena Di Napoli

Istituto Nazionale Tumori IRCCS Fondazione Pascale

Sabrina Rossetti

Istituto Nazionale Tumori IRCCS Fondazione Pascale

Egidio Celentano

Istituto Nazionale Tumori IRCCS Fondazione Pascale

Anna Crispo

Istituto Nazionale Tumori IRCCS Fondazione Pascale

Maria Grimaldi

Istituto Nazionale Tumori IRCCS Fondazione Pascale

Paolo Muto 


\section{Study protocol}

Keywords: Palliative care, metastatic disease, radiotherapy, cancer pain, Breakthrough cancer Pain, quality of life

Posted Date: March 18th, 2021

DOl: https://doi.org/10.21203/rs.3.rs-316250/v1

License: (a) (i) This work is licensed under a Creative Commons Attribution 4.0 International License. Read Full License 


\section{MAnagement of METastatic disease In Campania (MAMETIC): Epidemiologic Data and Palliative Radiotherapy in an Italian Region. Study Protocol}

Di Franco $\mathrm{R}^{1}$, Cascella $\mathrm{M}^{2}$, Fusco $\mathrm{M}^{3}$, Borzillo $\mathrm{V}^{1}$, Ferraioli $\mathrm{P}^{1}$, Scipilliti $\mathrm{E}^{1}$, D'Ippolito $\mathrm{E}^{1}$, Petito $\mathrm{A}^{1}$, Cuomo $\mathrm{A}^{2}$, Pignata $\mathrm{S}^{4}$, Di Napoli $\mathrm{M}^{4}$, Rossetti $\mathrm{S}^{4}$, Celentano $\mathrm{E}^{5}$, Crispo $\mathrm{A}^{5}$, Grimaldi $\mathrm{M}^{5}$, Muto $\mathrm{P}^{1}$

${ }^{1}$ Department of Radiation Oncology, Istituto Nazionale Tumori - IRCCS - Fondazione G. Pascale, Napoli

${ }^{2}$ Division of Anesthesia and Pain Medicine, Istituto Nazionale Tumori, IRCCS Fondazione G. Pascale, Napoli

${ }^{3}$ Cancer Registry Unit, ASL Napoli 3 SUD

${ }^{4}$ Department of Uro-Gynecological, Istituto Nazionale Tumori - IRCCS - Fondazione G. Pascale, Napoli

${ }^{5}$ Epidemiology and Biostatistics Unit, Istituto Nazionale Tumori - IRCCS - Fondazione G. Pascale, Napoli

Corresponding Author:

Di Franco Rossella - Department of Radiation Oncology, Istituto Nazionale Tumori - IRCCS - Fondazione G. Pascale, Napoli

r.difranco@istitutotumori.na.it 


\begin{abstract}
Background. In the Italian Campania Region, 30.517 new cases of solid cancer have been diagnosed, in 2019. Of those, patients with metastatic disease are up to $20 \%$. This class of patients is extremely diversified and copious, and the offer of radiotherapy may vary in different geographical areas within the same region. The aim of this observational multicenter retrospective and prospective trial is to evaluate the occurrence of metastatic disease in several areas of a great Italian region, the management of the disease through RT approaches, and its impact on cancer-related pain and overall HRQoL.
\end{abstract}

Methods. This is a multicenter, retrospective and prospective observational investigation. The retrospective part of the study concerns all patients enrolled with a diagnosis of metastatic disease and treated in RT centers within the Campania Region between January 2019 to July 2020. The prospective phase is going to involve all the metastatic patients with an indication of palliative RT. Considering regional epidemiological data, we expect an enrollment of 12.500-21.000 patients in 5 years.

Conclusion. The MAMETIC Trial is an epidemiological study designed for investigating the different prevalence of tumors in the metastatic phase within a regional area, and for evaluating the local response to the patient's request. It can be a unique opportunity, not only to highlight possible geographic differences but also to regularly collect and share data to standardize the therapeutic offer within the regional area. ClinicalTrials.gov ID NCT04595032, retrospectively registered.

Keywords: Palliative care, metastatic disease, radiotherapy, cancer pain, Breakthrough cancer Pain, quality of life

\title{
List of abbreviations
}

AIOM Association of Medical Oncology

ASIA American Spinal Injury Association

BTcP Breakthrough cancer pain

EORTC European Organization for Research and Treatment of Cancer

HRQoL Health-related quality of life

IMRT intensity-modulated radiation therapy

MAMETIC MAnagement of METastatic disease In Campania

NRS Numeric Rating Scale

RT Radiotherapy

SEER Surveillance, Epidemiology and End Results

SBRT stereotactic body radiation therapy

SINS Spinal Instability Neoplastic Score

3DCRT three-dimensional conformal radiation therapy

VMAT volumetric modulated arc therapy 


\section{Background}

The overall survival is the main outcome for evaluating healthcare system efficiency in cancer treatment. It is highly influenced by the diagnosis phase and cancer treatment efficacy. Secondary prevention, availability, and rapid access to treatments have a crucial influence.

Although it was demonstrated that the number of cancer deaths in 2005 and 2016 is roughly the same, the number of cancer deaths recorded with metastasis as a contributing cause of death tripled from $1953(18.5 \%)$ to $2016(55.5 \%)^{1}$. Furthermore, metastases are responsible for about $90 \%$ of cancer deaths worldwide ${ }^{2}$.

The proportion of all cancer deaths recorded with metastases ranged from $100 \%$ (testicular, sinus cancer) to $9.3 \%$ for central nervous system cancers. For prostate cancer, the cancer death rate recorded with metastasis as a contributing cause was only 50.5\%. Breast cancer incidence has increased over the past decade, while breast cancer deaths are declining. The death rate from metastatic disease, however, is remarkably stable ${ }^{2}$. In the Italian context, data from the Italian Association of Medical Oncology (AIOM) showed that for all solid tumors, $66.7 \%$ of cancer deaths were recorded with metastasis as a concomitant cause, although the proportions varied substantially between tumor groups ${ }^{3}$.

In metastatic disease, palliative care has the essential purpose to improve the health-related quality of life (HRQoL). The earliest diagnosis is provided, the greater will be the aims achieved and the efficacy of the treatments. The local presence of diagnostic and therapeutic possibilities can be decisive in the patient's perspective on life. Nevertheless, in some geographical contexts, there may be deficiencies in the provision of particular palliative care interventions, such as radiation therapy (RT) approaches. These deficiencies can be expressed in terms of reduced supply of procedures, delays in delivery, and poor quality of the offer. Moreover, within a geographical region, inequality in access to diagnostic-therapeutic pathways can be detected.

Territorial cancer registers give a fundamental contribution for evaluating the incidence of cancer, the stage at diagnosis, and the correlation with particular environmental situations. In the Italian Campania Region, the recording cancer network has provided a General Cancer Registry of the population within each Local Health and a Regional Children's Cancer Registry. Despite this, there is a lack of important data such as the prevalence of metastatic cancer, referring to the different territorial areas within the region.

\section{Study rationale}

From the data mainly obtained from the SEER (Surveillance, Epidemiology, and End Results) archive, it is estimated that patients with metastatic disease represent up to $20 \%$ of the total number of solid tumors diagnosed ${ }^{3}$. Furthermore, according to the latest AIOM report in 2019, and excluding non-melanoma skin cancers, the Campania Region diagnosed 30.517 new cases of solid cancer. Consequently, applying the United States (US) prevalence figure (20\%), the number of metastatic patients for solid tumors in Campania should be around 6.100 patients, per year ${ }^{4}$.

On these premises, the MAnagement of METastatic disease In Campania (MAMETIC) study aims to evaluate metastatic cancer patients living in the Italian Campania region who refer to RT centers for palliative radiation treatments. Moreover, the management of the disease through RT approaches and its impact on cancer-related pain and overall patients' HRQoL will be investigated. 
All the data will be transmitted to the coordinating center and the initiative will be integrated with the regional oncology network (the General Cancer Registry). We estimate that this study will be useful not only to the RT community but it will also provide a regional assessment on the management of malignant disease, enhancing in turn the functioning of the network.

\section{Methods}

\subsection{Overall design}

The MAMETIC Trial is a multicenter, retrospective and prospective observational investigation (Fig. 1).

a. The retrospective part of the study concerns all patients enrolled with a diagnosis of metastatic disease, treated in RT centers of the Campania Region. RT was performed through several approaches including three-dimensional conformal radiation therapy (3DCRT), intensitymodulated radiation therapy (IMRT), volumetric modulated arc therapy (VMAT), stereotactic body radiation therapy (SBRT). Treatments were performed from January 2019 to July 2020.

Because in 2019, about 12.500 patients have been treated in the centers and it is estimated that $20-30 \%$ of the above-mentioned patients had metastasis, approximately 2.560 patients are expected to be enrolled.

b. The prospective part of the study is going to involve all the metastatic patients with indication of palliative RT, treated with 3DCRT, IMRT, VMAT, SBRT techniques from December 2020 to December 2025. Considering regional epidemiological data, we expect an enrollment of patients between $2.500-4.200$ patients per year, 12.500-21.000 in 5 years.

\subsection{Study setting}

RT centers joining the study can enter patient data in appropriate eCRFs. An eCRF will be provided for the retrospective part of the study. About the prospective part, RT centers can choose 3 levels of membership:

- Level A: epidemiological data entry on clinical features (metastatic cancers) and RT offered.

- Level B: level A plus pain features and analgesic therapy data.

- Level C: adds to the two previous levels data on HRQoL.

We have chosen to offer a different membership option to collect the greatest number of epidemiological information (primary objective). From the centers that will be able to enter the most complete data (Level B and level C), it will be possible to obtain data to achieve the secondary objectives. 


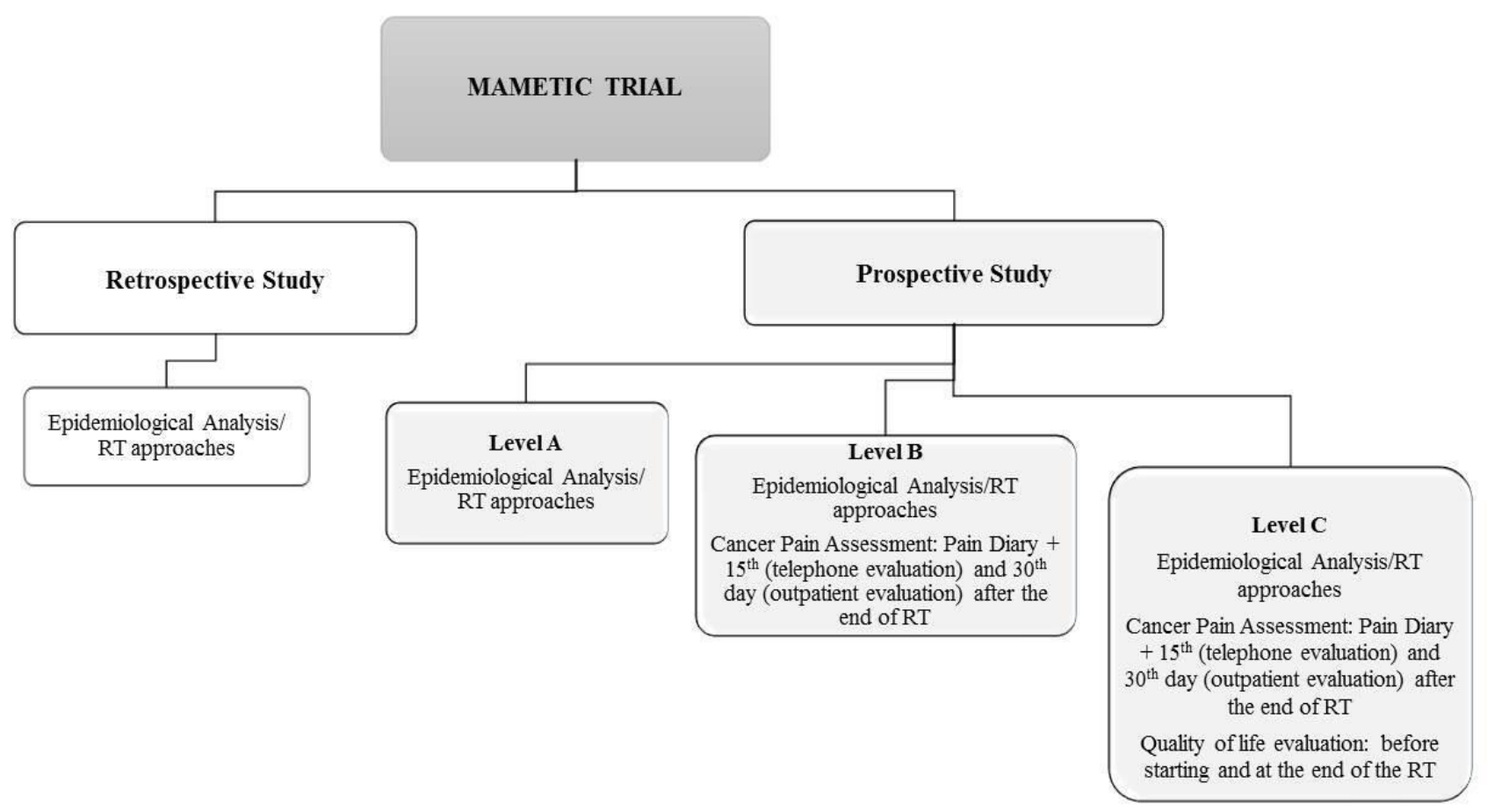

Fig.1. Study design flowchart. Legend: RT, radiation therapy

\subsection{Outcome measures}

The primary objective of the study is focused on the data collection concerning metastatic cancers in the Campania region. This objective regards both the retrospective and prospective phases of the study.

For this purpose, will be recorded:

- Epidemiological data (patient/disease):
○ Patient data
- Cancer type
- Metastasis
- Incidence of metastatic disease at diagnosis
- Time between first diagnosis and the onset of metastases

Secondary objectives of the study are:

- Characterization of palliative RT offered

- Cancer pain features

- Patients' HRQoL 


\subsection{Study population}

Eligible candidates for MAMETIC are metastatic patients candidates for palliative RT.

- Patients with bone metastases. They are about $70 \%$ of cancer patients in advanced stage of disease 5 . They often have complicated lesions, with tumor tissue invading the muscle or nerve structures close to the bone. These lesions usually cause constant or intermittent pain, with a neuropathic component. In addition to baseline pain, the patient may have episodes of BTcP (Breakthrough cancer Pain) that compromise the HRQoL. As a result, they may have severe movement restrictions and an increased risk of pathological bone fracture ${ }^{6-9}$.

- Patients with metastases affecting the thoracic district with primary or secondary locally advanced disease. Symptoms such as dyspnea, chest pain, cough, hemoptysis, and dysphagia can be controlled with radiation treatments in $2 / 3$ of these patients. Palliative mediastinal RT can improve obstructive dysphagia in locally advanced esophageal cancer ${ }^{10}$. At the same time, it can improve esophageal function after insertion of a stent ${ }^{11}$. Again, in patients with mediastinal syndrome, radiant treatment of the whole or part of mediastinum can ameliorate the HRQoL.

- Patients with bleeding, who may have neoplastic bleeding in the gastrointestinal, genitourinary, upper respiratory tract, or pelvic tract. For these patients, RT can have an important hemostatic effect and consequently can improve symptoms and hematological values. Radiant treatment can be used for hemostatic purposes in patients with bladder, rectal or gynecological malignancies with an improvement of the symptoms in 50-75\% of cases.

- Patients with brain or spinal cord metastases. This category includes patients with symptomatic or asymptomatic brain metastases, patients with symptomatic or asymptomatic bone marrow compression, and those with cauda equina syndrome. In patients with paraplegia caused by spinal cord compression, $10 \%$ of motility can be recover if treatment is early. The presence of brain metastases can cause severe symptoms such as epilepsy, focal neurological symptoms, or intracranial hypertension symptoms such as nausea, vomiting, or headache ${ }^{12}$.

- Patients with symptomatic lymph node lesions who may have neck lymph node packages or pelvic district.

Eligibility criteria are summarized in Table 1

\begin{tabular}{|c|c|}
\hline Table 1. MAMETIC Trial Eligibility criteria \\
\hline Inclusion criteria & Exclusion criteria \\
\hline$\cdot \begin{array}{l}\text { Patients aged } \geq 18 \text { years resident in the } \\
\text { Campania Region }\end{array}$ & $\cdot$ Patients aged <18 years \\
\hline$\cdot \begin{array}{l}\text { Patients diagnosed with metastatic disease } \\
\text { candidates for radiotherapy }\end{array}$ & $\begin{array}{l}\text { Patients who are unable to express consent } \\
\text { to sensitive data and radiant treatment }\end{array}$ \\
\hline$\cdot \begin{array}{l}\text { Metastatic patients candidates for } \\
\text { radiotherapy re-treatment }\end{array}$ & \\
\hline$\cdot \begin{array}{l}\text { Oligometastatic patients candidates for } \\
\text { radiotherapy }\end{array}$ & \\
\hline$\cdot$ & Patients who have given their consent \\
\hline
\end{tabular}




\subsection{Clinical Variables}

Clinical characteristics of patients will be collected from hospital clinical documentation and RT treatment plans, according to data needed for the study. Data will be identified in the medical record and collected in specific eCRF:

\section{A. RETROSPECTIVE STUDY:}

- Address

- Weight

- Height

- Primary tumor

- Date of the first diagnosis

- Stage c / pTNM at diagnosis

- Therapies carried out for the primary tumor (surgery, chemotherapy, hormone therapy, immunotherapy, target therapies, RT)

- Metastatic disease diagnosis date

- Pre-treatment radiotherapy visit date

- Start and end date of palliative RT

- Possible request for rapid access to RT

- Indications for palliative RT (bone marrow compression, mediastinal syndrome, hemostasis, bone metastasis painful, painless bone metastasis, compression or infiltration pain, bone - lung - liver - lymph node - adrenal oligometastasis, brain metastases, primitive encephalic with expired PS)

- Type of treatment: RT flash, SBRT

- Prescribed dose and fractions

- Re-treatment.

\section{B. PROSPECTIVE STUDY}

a. Level A. The same epidemiological/RT data recorded in the retrospective study

b. Level B: Epidemiological/RT data plus:

- Type of pain (neuropathic, nociceptive, mixed) and intensity measured through the Numeric Rating Scale (NRS) score. This latter is an 11-point scale (from 0 to 10 ), with higher scores indicating greater pain intensity.

- Breakthrough cancer pain (BTcP) [defined as 'a transitory flare of pain that occurs on a background of relatively well-controlled baseline pain' $\left.{ }^{13}\right]$ in terms of number of episodes/day; pain intensity ${ }^{14}$.

- Pain flare (a transient worsening of the bone pain after RT) in terms of intensity (NRS).

- Data about pain therapy: drugs (adjuvant drugs, opioids, non-opioid analgesics) and doses.

- Adverse events of the pain therapy.

- For the pain evaluation, patients will report pain data (NRS, episodes of BTcP) and therapy in a Pain Diary. Follow-ups will be performed on the $15^{\text {th }}$ 
(telephone revaluation) and $30^{\text {th }}$ day (outpatient revaluation) after the end of RT treatment.

c. Level C. In addition to what is specified for levels A and B, the following tools for assessing the patients' HRQoL will be adopted before starting and at the end of the RT treatment:

- The European Organization for Research and Treatment of Cancer (EORTC) QLQC15-PAL questionnaire ${ }^{15}$, and Palliative Performance Score (PPS) ${ }^{16}$ for all patients.

- The EORTC QLQ-BM22 ${ }^{17}$ questionnaire; van der Linden Score ${ }^{18}$; Mizumoto Prognostic Score ${ }^{19}$ for assessing the HRQoL in patients with bone metastases.

- The Spinal Instability Neoplastic Score (SINS) ${ }^{20}$ used for investigating the incidence of spinal instability in patients with spinal metastases.

- The American Spinal Injury Association (ASIA) Score ${ }^{21}$ for evaluation of the functional impairment as a result of the spinal cord injury/compression.

- The Mini-Mental State Examination $(\mathrm{MMSE})^{22}$ for assessing cognitive function in patients with brain metastases.

\subsection{Data collecting and Statistical considerations}

The data collection and registration procedures will be carried out through specific CRFs organized on an electronic platform managed by the coordinating center which will be responsible for the privacy of sensitive data. The platform was created with a management software developed with Microsoft Net technology, Web App made with Internet Information Services (IIS 10) pattern, MVC, Razor syntax, Jquery 3.4.1 scripting language. Net framework version 4.8. SQL Server 2019 database version 15. Crystal Reporting for Visual Studio 2019.

The data will be transmitted to the coordinating center when each form is completed by the operator of the individual center. Every 3 months a report of the total amount of patients entered in the platform will be sent by the coordinating center to every center participating in the study.

A descriptive analysis will summarize the total number and stratification of patients with metastatic disease by place of residence and tumor location. Metastatic tumors at diagnosis will be differentiated from metastatic disease developed later. Every center is going to fill a form for each patient with mandatory socio-demographic and anamnestic information (Level A) and information on pain and HRQoL (Levels B and C). This process lets the study produce a compliance survey by a single center and carry on a comparative analysis for subsequent levels on qualitative variables (presence of pain; typology and characterization of pain) through the Chi-Square Test and quantitative variables (pain scale, scores on quality of life) with T-test and ANOVA. Nonparametric tests will be provided where the population distribution does not have a known form (normal Gaussian). The significance of the tests is fixed with $p<0.05$.

\subsection{Ethics considerations}


The MAMETIC Trial is promoted by the Division of Radiotherapy of National Cancer Institute IRCCS Fondazione Pascale, Naples (Italy), in collaboration with the Divisions of Anesthesia and Pain Medicine, Uro-Gynecological Medical Oncology, Epidemiology and Biostatistics of the Fondazione Pascale, and with the Cancer Registry Unit ASL-Napoli 3 South. The local Ethics Committee approved the protocol on July 8, 2020 (n. 868/2020).

Each interested center will submit the protocol to its ethics committee to be approved before accrual. After approval, the center will receive a dedicated electronic case report form (CRF). Eligible patients who provided consent and meet the inclusion criteria are anonymously registered on the CRF by assigning a numerical code.

The Trial will be conducted in accordance with the approved protocol and the Helsinki Declaration (1996), the Guidelines for Good Clinical Practice CPM / ICH135 / 95-DM 15/7/97, in compliance with Legislative Decree n. 200 of 6 November 2007, Implementation of Directive 2005/2 // EC Article 3 and GDPR EU Regulation no. 2016/679.

\section{Discussion}

Palliative RT is an efficient component of supportive and palliative care in the oncology field and palliation of advanced and metastatic cancers symptoms are one of the most common indications for RT.

Although it was estimated that patients with metastatic disease represent up to $20 \%$ of the total number of solid tumors diagnosed, the use of palliative RT treatments potentially involves only up to $40 \%$ of patients in a radiation oncology center ${ }^{22}$.

The oncological incidence data, detected by population risk and collected from the Cancer registries, show different standardized rates in various regional territorial areas. Moreover, at the regional level, data on territorial distribution and evolution of tumors towards the advanced stages are absent. In addition to oncological mortality data, this knowledge could occur significantly to understand the causes of the several territorial possibilities in the organization of diagnostic-therapeutic-care pathways in terms of socio-economic structure of reference population, degree of diagnostic anticipation and different stage at diagnosis, availability of territorial diagnosis and treatment structures, level of care standards. Furthermore, there is a lack of precise knowledge on coverage and methods of RT services regional delivery in the advanced stages of neoplastic pathology in Campania.

\section{Conclusion}

A regional epidemiological study that provides information on the occurrence of late-stage tumors, their territorial management, and their impact on the HRQoL of patients, can represent an important opportunity to observe territorial alternatives and improve the therapeutic offer. At the same time, since this is a multicenter study involving several RT centers, it can represent a useful moment of standardization and sharing of RT protocols adopted in the treatments of advanced tumors and their complications in a great Italian region.

\section{Funding}

The trial will be conducted without external funding.

\section{Authors' contributions}


RDF, PM, MC, AC, MF conceived the study and led the protocol development; DA, VB, EDI, ES, MG, SP, MDN, AP, contributed to the development of the study protocol. AC, and EC (lead trial statistician) developed the study design and statistical analysis plan. All authors provided feedback on drafts of this paper and read and approved the final manuscript.

\section{Consent for publication}

The authors consent to data's publication

\section{Availability of data and material}

The authors declare the availability of data and material

\section{Competing interests}

The authors declare that they have no competing interests.

\section{Acknowledgements}

The authors gratefully acknowledge Mrs. Diana d'Alterio for the contribute in the manuscript editing and Mr. Rocco Saviano for the technical support in creating the electronic platform

\section{References}

1. Dillekås H, Rogers MS, Straume O - Are $90 \%$ of deaths from cancer caused by metastases? Cancer Med. 2019;8(12):5574-5576.

2. Dillekas H, Demicheli R, Ardoino I, Jensen SA, Biganzoli E, Straume O. The recurrence pattern following delayed breast reconstruction after mastectomy for breast cancer suggests a systemic effec of surgery on occult dormant micrometastases. Breast Cancer Res Treat. 2016;158(1):169-78.

3. Italian Association of Medical Oncology. AIOM-AIRTUM. [Data on cancer in Italy, 2019]. In Italian. Brescia: Intermedia Editore, 2020. Available at: https://www.aiom.it/wp-content/uploads/2019/09/2019 Numeri Cancrooperatori-web.pdf. Last accessed, November 4, 2020.

4. Huang JF, Shen J, Li X, Rengan R, Silvestris N, Wang M, Derosa L, Zheng X, Belli A, Zhang XL, Li YM, Wu M. Incidence of Patients With Bone Metastases at Diagnosis of Solid Tumors in Adults: A Large Population-Based Study Ann Transl Med. 2020;8(7):482. doi: 10.21037/atm.2020.03.55

5. Coleman RE. Clinical features of metastatic bone disease and risk of skeletal morbidity. Clin Cancer Res. 2006;12:6243s-9s. 10.1158/1078-0432.CCR-06-0931 17062708

6. Di Franco R, Falivene S, Ravo V, Mammucari M, Sarli E, Baffini S, De Palma G, Pepe A, Traettino M, Muto M, Cappabianca S and Muto P. Management Of Painful Bone Metastases: Our Experience According To Scientific Evidence On Palliative Radiotherapy. Anticancer Research. 2004;34:1011-14.

7. Falivene S, Pezzulla D, Di Franco R, Giugliano FM, Esposito E, Scoglio C, Amato B, Borzillo V, D'Aiuto M, Muto P. Painful bone metastasis in elderly treated with radiation therapy: Single- or multiple-fraction regimen? A multicentre retrospective observational analysis. Aging Clin Exp Res. 2017 Feb;29(Suppl 1):143-147.

8. Giugliano FM, Franco RD, Iadanza L, Borzillo V, Cutillo L et al. (2014) Elderly Patients with Painful Bone Metastases. The Impact of Comorbidity on the Choice of Radiation Therapy Regimen. J Palliat Care Med 4:199. doi:10.4172/2165-7386.1000199

9. Di Franco R, Falivene S, Ravo V, Borzillo V, Giugliano FM, Argenone A, Rossetti S, Cavaliere C, D'aniello C, Romano FJ, Berretta M, Facchini G, Muto P. Impact of Procedural Pain In Radiotherapy Treatment WCRJ 2017; 4 (2): e884

10. Kassam Z, Wong RKS, Ringash J, Ung Y, Kamra J, DeBoer G, O'Brien M, Kim J, Loblaw DA, Wong S, Cummings B, Davey P - A phase I/II study to evaluate the toxicity and efficacy of accelerated fractionation 
radiotherapy for the palliation of dysphagia from carcinoma of the oesophagus. Clin Oncol (R Coll Radiol). 2008;20:53-60.

11. Javed A, Pal S, Dash NR, Ahuja V, Mohanti BK, Vishnubhatla S, Sahni P, Chattopadhyay TK - Palliative stenting with or without radiotherapy for inoperable esophageal carcinoma: a randomized trial. J Gastrointest Cancer. 2012;43:63-9.

12. Borzillo V, Giugliano FM, Di Franco R, Falivene S, Cammarota F, Ravo V, Muto P. Radiosurgery and Stereotactic Radiotherapy for Brain Metastases According the New Prognostic Indexes: our Preliminary Experience Neurol Neurophysiol 2015, 6:6

13. Portenoy RK, Hagen NA. Breakthrough pain: definition, prevalence, and characteristics. Pain. 1990; 41: 273281.

14. Cuomo A, Cascella M, Forte CA, et al. Careful Breakthrough Cancer Pain Treatment through Rapid-Onset Transmucosal Fentanyl Improves the Quality of Life in Cancer Patients: Results from the BEST Multicenter Study. J Clin Med. 2020;9(4):1003. Published 2020 Apr 2. doi:10.3390/jcm9041003

15. Lam K, Zeng L, Zhang L, Tseng LM, Hou MF, Fairchild A, Vassiliou V, Jesus-Garcia R, El-Din MAA, Kumar A, Forges F, Chie WC, Sahgal A, Poon M, Chow E. Predictive factors of overall well-being using the EORTC QLQ-C15-PAL extracted from the EORTC QLQ-C30. J Palliat Med. 2013; 16(4):402-8.

16. Yoon SJ, Choi SE, LeBlanc TW, Suh SY. Palliative Performance Scale Score at 1 Week After Palliative Care Unit Admission is More Useful for Survival Prediction in Patients With Advanced Cancer in South Korea. Am J Hosp Palliat Care. 2018;35(9):1168-1173.

17. Mendez LC, Raman S, Wan BA, da Silva JLP, Moraes FY, Lima KMLB, Silva MF, Diz MDPE, Chow E, Marta GN. Quality of life in responders after palliative radiation therapy for painful bone metastases using EORTC QLQ-C30 and EORTC QLQ-BM22: results of a Brazilian cohort. Ann Palliat Med. 2017;6(Suppl 1):S65-S70

18. van der Linden YM, Dijkstra SPDS, Vonk EJA, Marijnen CAM, Leer JWH. Dutch Bone Metastasis Study Group. Prediction of survival in patients with metastases in the spinal column: results based on a randomized trial of radiotherapy. Cancer. 2005;103(2):320-8.

19. Mizumoto M, Harada H, Asakura H, Hashimoto T, Furutani K, Hashii H, Takagi T, Katagiri H, Takahashi M, Nishimura T. Prognostic factors and a scoring system for survival after radiotherapy for metastases to the spinal column: A review of 544 patients at Shizuoka Cancer Center Hospital. Cancer. 2008;113(10):2816-22.

20. Shi DD, Hertan LM, Lam TC, Skamene S, Chi JH, Groff M, Cho CH, Ferrone ML, Harris M, Chen YH, Balboni TA. Assessing the utility of the spinal instability neoplastic score (SINS) to predict fracture after conventional radiation therapy (RT) for spinal metastases. Pract Radiat Oncol. 2018;8(5):e285-e294.

21. van Middendorp JJ, Goss B, Urquhart S, Atresh S, Williams RP, Schuetz M. Diagnosis and Prognosis of Traumatic Spinal Cord Injury. Global Spine J. 2011; 1(1): 1-8.

22. Murray KJ, Scott C, Zachariah B, Michalski JM, Demas W, Vora NL, Whitton A, Movsas B. Importance of the mini-mental status examination in the treatment of patients with brain metastases: a report from the radiation therapy oncology group protocol. International Journal of Radiation Oncology Biology Physics. 2000;48(1):5964.

23. Cellini F, Manfrida S, Deodato F, Cilla S, Maranzano E, Pergolizzi S, Arcidiacono F, Di Franco R, Pastore F, Muto M, Borzillo V, Donati CM, Siepe G, Parisi S, Salatino A, D'Agostino A, Montesi G, Santacaterina A, Fusco V, Santarelli M, Gambacorta MA, Corvò R, Morganti AG, Masiello V, Muto P, Valentini V. Pain REduction with bone metastases STereotactic radiotherapy (PREST): A phase III randomized multicentric trial. Trials. 2019;20(1):609. 


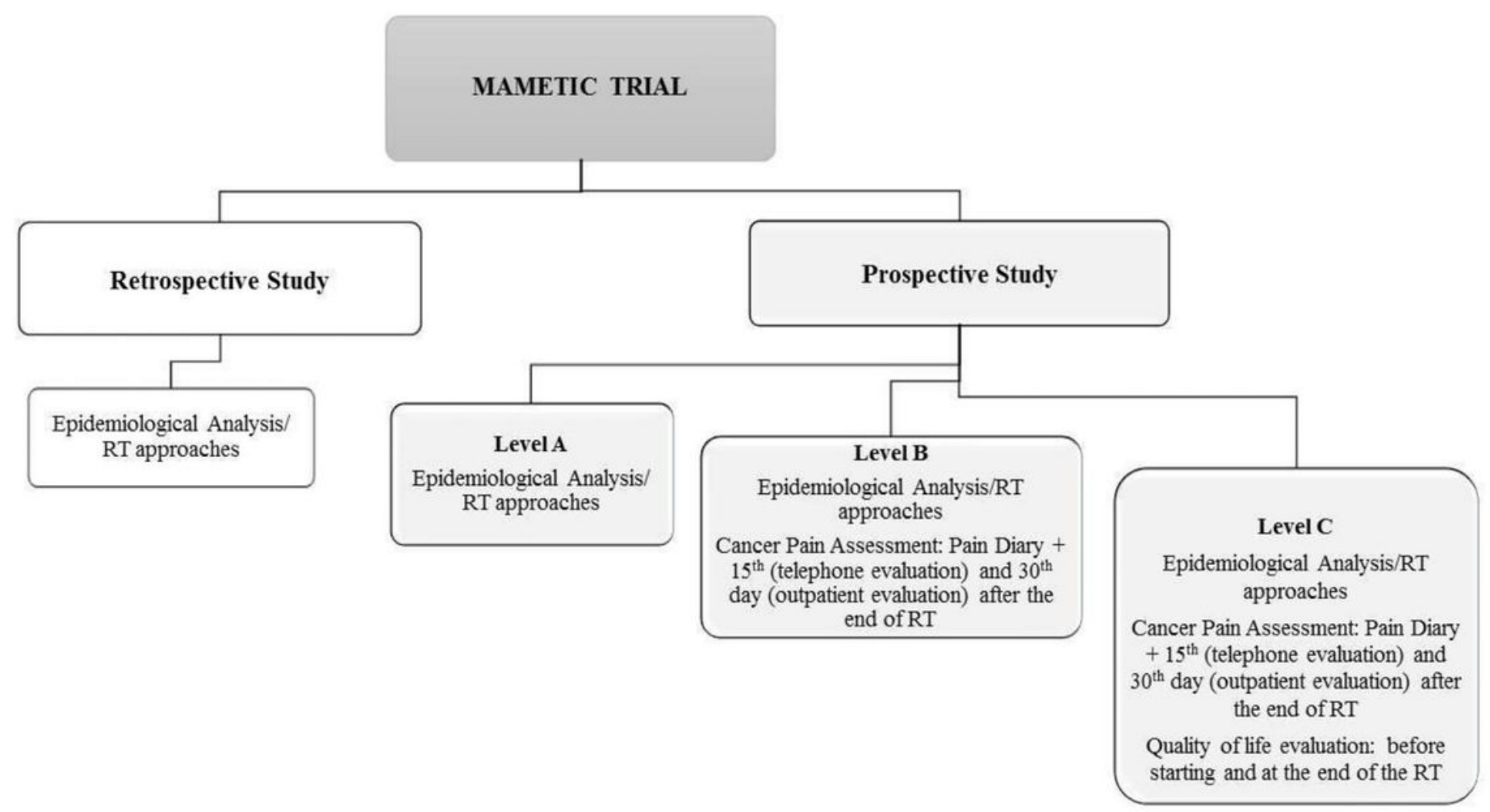

Figure 1

Study design flowchart. Legend: RT, radiation therapy 